\title{
Numerical Bifurcation Analysis of Marine Ice Sheet Models
}

\author{
T. E. Mulder, H. A. Dijkstra and F. W. Wubs
}

\begin{abstract}
The climate variability associated with the Pleistocene Ice Ages is one of the most fascinating puzzles in the Earth Sciences still awaiting a satisfactory explanation. In particular, the explanation of the dominant $100 \mathrm{kyr}$ period of the glacial cycles over the last million years is a long-standing problem. Based on bifurcation analyses of low-order models, many theories have been suggested to explain these cycles and their frequency. The new aspect in this contribution is that, for the first time, numerical bifurcation analysis is applied to a two-dimensional marine ice sheet model with a dynamic grounding line. In this model, we find Hopf bifurcations with an oscillation period of about $100 \mathrm{kyr}$ which may be relevant to glacial cycles.
\end{abstract}

Keywords: Marine Ice Sheets, Bifurcation Analysis, Multiple Equilibria, Oscillatory Modes

\section{Introduction}

Very detailed information on past temperatures on Earth has been obtained from marine benthic records, in particular oxygen isotope ratios. Water in ice cores contains two isotopes of oxygen, ${ }^{18} \mathrm{O}$ and ${ }^{16} \mathrm{O}$. The normalized isotope ratio $\delta^{18} \mathrm{O}$ is calculated as a deviation from a reference sample as

T. E. Mulder and H. A. Dijkstra

Institute for Marine and Atmospheric research Utrecht, Department of Physics, Utrecht University, the Netherlands, e-mail:h.a.dijkstra@uu.nl

F. W. Wubs

Johann Bernoulli Institute for Mathematics and Computer Science, Groningen University, Groningen, The Netherlands e-mail: f.w.wubserug.nl 


$$
\delta^{18} O=\frac{\left(\frac{18 O}{16}\right)_{\text {sample }}-\left(\frac{18 O}{16}\right)_{\text {reference }}}{\left(\frac{18 O}{1{ }^{16} O}\right)_{\text {reference }}},
$$

where the reference sample is 'standard mean' ocean water. The isotope ${ }^{16} \mathrm{O}$ is lighter than ${ }^{18} \mathrm{O}$ so that water containing ${ }^{16} \mathrm{O}$ is preferentially evaporated and a temperature-dependent fractionation occurs. Changes in $\delta^{18} O$ reflect the combined effect of changes in global ice volume and temperature at the time of deposition of the sampled material. During very cold conditions, global ice volume is relatively large and hence sea level is low, which enriches water in the ocean with ${ }^{18} \mathrm{O}$. Also because of the colder temperatures, more ${ }^{18} \mathrm{O}$ remains in the ocean and less ${ }^{18} \mathrm{O}$ becomes locked in the ice. Hence, the ratio $\delta^{18} O$ in ice cores will decrease (becomes more negative) under colder conditions.

In marine sediment cores the opposite behavior of the isotope ratio is found and $\delta^{18} O$ increases (becomes more positive) when the temperature decreases (during colder conditions, the concentration of the heavier isotope will increase). In Fig. 1, a time series is shown of a composite $\delta^{18} \mathrm{O}$ ocean sediment (benthic) record over the last $2 \mathrm{Myr}$ [1]. A cooling trend is found on which variability in ice cover is superposed. Analysis reveals that this variability is first dominated by a $41 \mathrm{kyr}$ period and after the so-called Mid Pleistocene Transition (MPT) at about $700 \mathrm{kyr}$, it is dominated by a $100 \mathrm{kyr}$ period.

The European Project for Ice Coring in Antarctica (EPICA) has provided two deep ice cores in East Antarctica from which climate conditions can be reconstructed back to $800 \mathrm{kyr}$ BP [2]. From the reconstructed temperature anomaly time series (Fig. 2), one observes the asymmetry between the slow glaciation and the rapid deglaciations. Glacial-interglacial transitions have affected all components of the climate system and induced relatively large amplitude changes of many variables in these components. One important player in the climate system responsible for the globalization of these transitions is believed to be the atmospheric $\mathrm{CO}_{2}$ concentration. A composite $\mathrm{CO}_{2}$ record is also shown in Fig. 2. created from a combination of records from the Dome $\mathrm{C}$ and Vostok ice cores. It is observed that the atmosphere $\mathrm{CO}_{2}$ concentration varies from about $180 \mathrm{ppm}$ to $280 \mathrm{ppm}$ during a glacial-interglacial transition and that an optimal correlation with the $\delta^{18} \mathrm{O}$ time series occurs near lag zero.

These results lead to many intriguing questions: Why did glacial-interglacial cycles appear in the Pleistocene? Which processes in the climate system caused the glacial-interglacial changes in global mean temperature and ice sheet extent? What caused the transition (the MPT) from the $41 \mathrm{kyr}$ world to the $100 \mathrm{kyr}$ world about 700 kyr ago?

Approaches to answers on the Pleistocene Ice Ages problem have a very interesting history which is nicely described in [3]. A connection with the orbital characteristics of the Earth-Sun system was already made in the $19^{\text {th }}$ century, but in the 1930s it was suggested [4] that glaciations occur when the insolation intensity is weak at high northern latitudes during summer. When the $65^{\circ} \mathrm{N}$ insolation is small, ice can persist throughout the year leading to the growth of ice sheets. Favorable conditions 


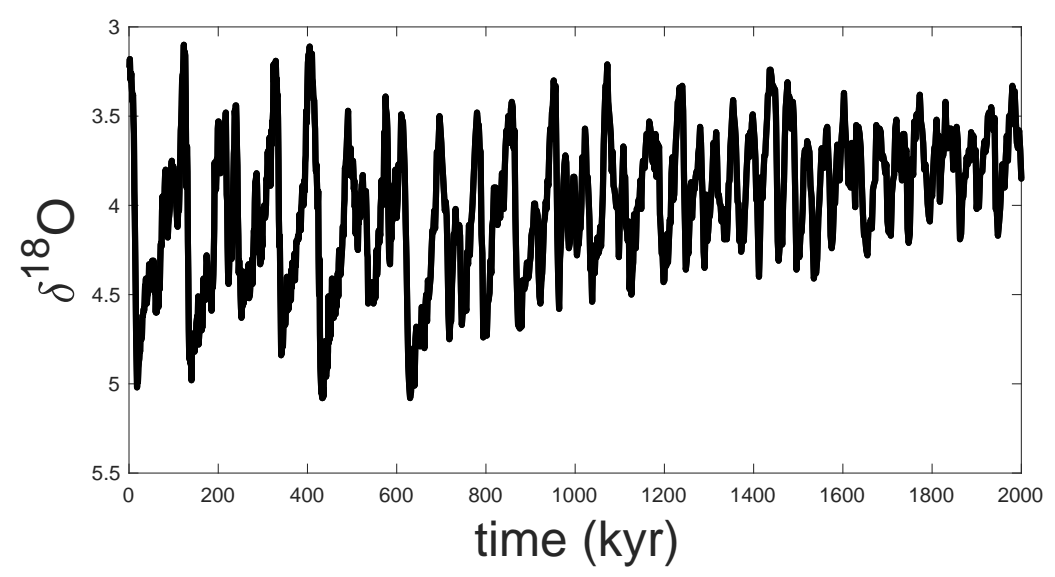

Fig. 1 (a) The LR04 benthic $\delta^{18} O$ stack over the Pleistocene, constructed by the graphic correlation of 57 globally distributed benthic $\delta^{18} O$ records (data from [1]). The $x$-axis indicates time $B C$ in kyr (so the time is increasing from right to left).

for this to happen are when the spin axis is less tilted and the aphelion (the point in the orbit, where the Earth is farthest from the Sun) coincides with summer in the Northern Hemisphere.

The variations in insolation are caused by the changes in orbital characteristics of the Earth, and there are three types of motion relevant for the amount of radiation received at a particular point on Earth. First, the spin axis of the Earth undergoes precession. One full cycle of precession has a period of $27 \mathrm{kyr}$, but coupled to the movement of the long end of the ellipse around the Sun (in $105 \mathrm{kyr}$ ) the net effect is a fluctuation in solar radiation with a period of $23 \mathrm{kyr}$. In addition, both the obliquity and the eccentricity of the Earth's orbit undergo periodic variations. The tilt angle changes in $41 \mathrm{kyr}$ between $22^{\circ}$ and $24^{\circ}$ leading to variations in seasonal contrast, and the eccentricity varies from 0.0 (perfect circle) to about 0.05 with periodicities of $100 \mathrm{kyr}$ and $450 \mathrm{kyr}$. A time series of the insolation at $60^{\circ} \mathrm{N}$ (Fig. 3 $\mathrm{k}$ ) clearly shows variations of about $100 \mathrm{Wm}^{-2}$ over the last $1 \mathrm{Myr}$. A comparison of the spectrum of this insolation curve (Fig. 3b) and the spectrum of a $\delta^{18} O$ record from an ocean sediment core (Fig. 3 $\mathrm{d}$ ) over the last $1 \mathrm{Myr}$ (as shown in Fig. 3k) shows that there are clear signatures of the 19 and $23 \mathrm{kyr}$ precession and of the $41 \mathrm{kyr}$ obliquity variations of the Earth's orbit in the Ocean Drilling Program record. On the other hand, at the $100 \mathrm{kyr}$ time scale, there is hardly any forcing amplitude while 


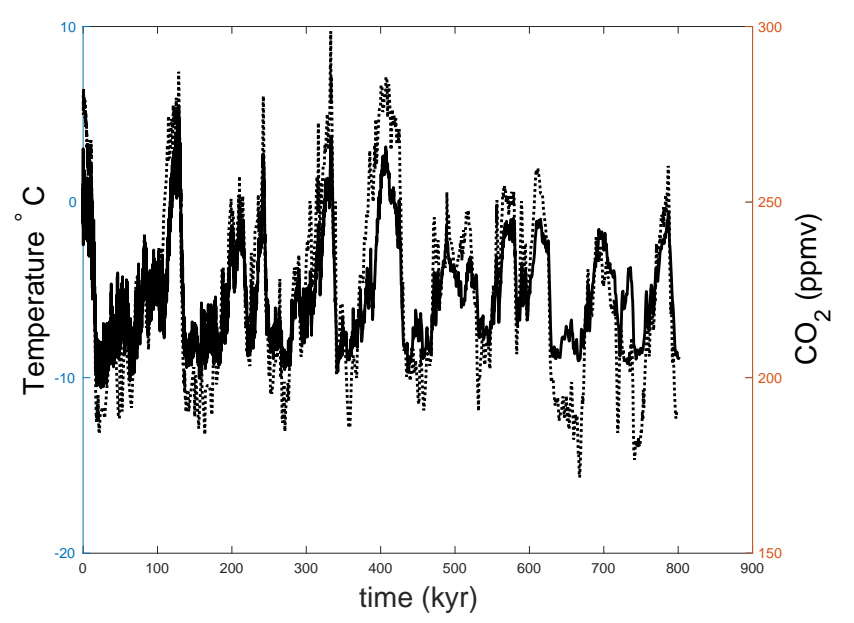

Fig. 2 Reconstructed temperature (drawn) and atmospheric $\mathrm{CO}_{2}$ (dotted) concentration from ice cores on Antarctica.

the climate signal in the $\delta^{18} O$ record has the largest amplitude. Comparing $\delta^{18} \mathrm{O}$ records with the insolation time series [5], it is interesting that the amplitude of the ice-cover variations can be very large while the insolation variation is very small. Furthermore, the variations in insolation provide no clue on the transition from the $41 \mathrm{kyr}$ world to the $100 \mathrm{kyr}$ world as they have the same temporal characteristics through the transition.

Although it is clear that the orbital insolation variations must play a role, a simple linear forcing-response relation apparently does not apply. The 100 kyr variations in insolation due to eccentricity are very weak (and it is the only forcing with an annual mean signal) and the $41 \mathrm{kyr}$ and $23 \mathrm{kyr}$ provide only low-frequency variations on the seasonal variations, not on the annual mean insolation. Hence, processes internal to the climate system must play a role in the amplification of the orbitally induced insolation variations.

\section{Basic theories of interglacial-glacial cycles}

There have been many suggestions on the dominant mechanisms of ice-age variability. The orbital variations in insolation over the globe are at the heart of all these theories. Traditionally, the June insolation at $65^{\circ} \mathrm{N}$ (such as shown in Fig. 3a) has been used as the most important part of this forcing as this determines whether snow will be left at the end of the Northern Hemisphere Summer season. Below, we will refer to this orbital component of the insolation as the M-forcing. 


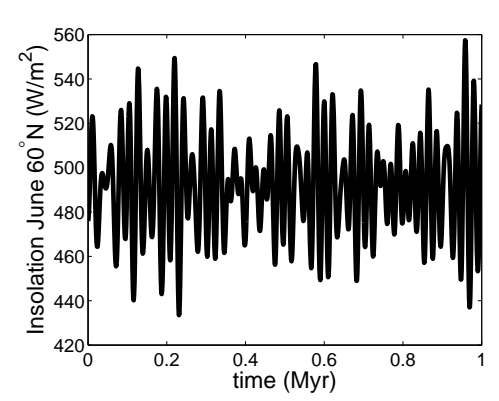

(a)

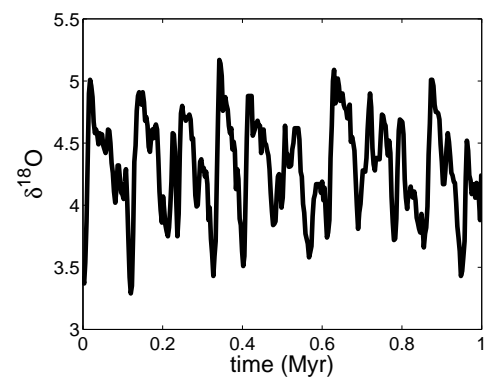

(c)

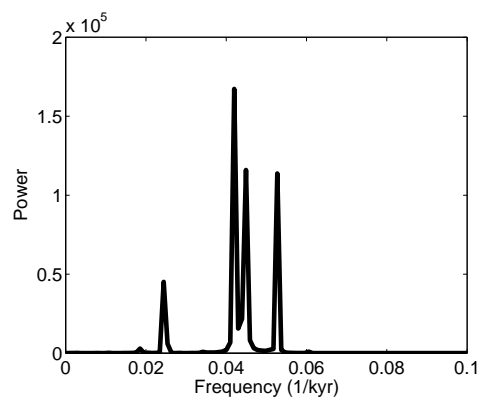

(b)

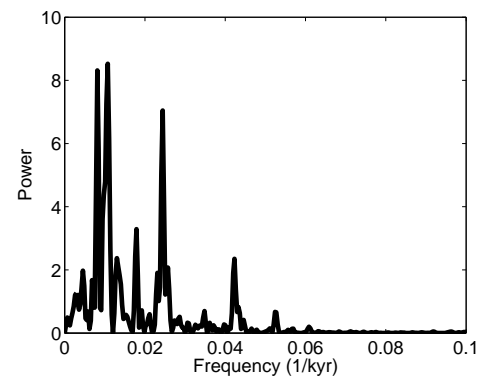

(d)

Fig. 3 (a) June insolation at $60^{\circ} N$. (b) Spectrum of the time series in (a). (c) Time series of $\delta^{18} \mathrm{O}$ at ODP677 $\left(83^{\circ} \mathrm{W}, 1^{\circ} \mathrm{N}\right)$ over the last $1 \mathrm{Myr}$. (d) Spectrum of the time series in (c). Note that $1 / 23=0.043,1 / 19=0.053,1 / 41=0.024$ and $1 / 100=0.01$.

In a first theory, the behavior of the climate system is considered as a transient deviation from a single steady equilibrium due to the $\mathrm{M}$-forcing. One can calculate that a $1 \%$ change in solar insolation leads to about a $1^{\circ} \mathrm{C}$ temperature change. The dominant amplitude of eccentricity variations is at a period of about $400 \mathrm{kyr}$ while the next strongest variations occur on about a $100 \mathrm{kyr}$ time scale. The variations in eccentricity do modify the globally and annually-averaged amount of insolation but the amplitudes are very small, in the order of $0.1 \%$ of the solar constant. The variations in eccentricity can therefore only account for a climate signal of at most 0.1 $\mathrm{K}$. A similar analysis gives that the total variations in precession and obliquity can only account directly for a signal of at most $0.5^{\circ} \mathrm{C}$, about an order of magnitude too small. When considering many other processes (e.g., ice sheets, bedrock) in the direct response to the $\mathrm{M}$-forcing, the sensitivity does not increase enough to explain the climate signal [6].

There have been several suggestions that the existence of multiple steady states gives, together with the M-forcing, rise to glacial cycles. For example, in the model by [7] there are three equilibrium states in the climate system: an interglacial state $\mathbf{i}$, a weak glacial state $\mathbf{g}$, and a strong glacial state $\mathbf{G}$. Transitions from $\mathbf{i}$ to $\mathbf{g}$ occur when the summer insolation at $65^{\circ} \mathrm{N}$ drops below a value $i_{0}$. Furthermore, transitions from $\mathbf{g}$ to $\mathbf{G}$ occur when the ice volume $V$ increases above some critical level 
$V_{c}$. Finally, a transition from $\mathbf{G}$ to $\mathbf{i}$ occurs when the insolation increases above a level $i_{1}$. These are the only transitions which are allowed in this model. By forcing the model with the M-forcing, there is a good overall agreement between model and observations (considering the simplicity of the model). By allowing for a slight linear trend in the maximum ice volume and one in the insolation forcing, [7] also finds the MPT at around the correct time and the spectra of his model and typical $\delta^{18} O$ data correspond reasonably well to each other.

When multiple equilibria, a weak periodic forcing, and noise are present there is also the possibility of stochastic resonance. In fact, the discovery of stochastic resonance actually occurred [8, 9, 10] while trying to explain the $100 \mathrm{kyr}$ dominant glacial cycles using an energy balance atmosphere model. The central element of this theory is the amplification of the weak periodic eccentricity component of the M-forcing by noise in the presence of multiple equilibria.

Coupled atmosphere-cryosphere and cryospheric-lithosphere processes can also give rise to internal variability. The question is of course, whether these processes can also give rise to sustained oscillations (through Hopf bifurcations). If so, the time scales and amplitude ranges of these oscillations with 'realistic' values of the parameters will be of interest regarding the glacial cycles. Typical results can be found in [11] where a sustained oscillation is indeed present under steady forcing. The time scale of the oscillation is about 6-7 kyr and not $100 \mathrm{kyr}$ which shows that the processes captured in this simple model are not able to generate this long time scale. In [12], the model in [13] is forced by an M-type forcing and the $100 \mathrm{kyr}$ period arises due to nonlinear resonances of the external frequency of the forcing and the internal frequency of oscillation [6].

Many other idealized models have been proposed which involve other components of the climate system [14], for example, those involving the atmospheric concentration of $\mathrm{CO}_{2}$ and the global ocean state. The model proposed by [15] attributes to the Antarctic ice sheet extent a central role in linking climatic and $\mathrm{CO}_{2}$ glacialinterglacial changes. The model proposed by [16] investigates the role of marine calcifiers in glacial-interglacial cycles. For many of these models, bifurcation diagrams have been computed showing that oscillations are associated with Hopf bifurcations [17].

A relatively simple model, where an internal oscillation exists with a time scale of about $100 \mathrm{kyr}$ is that of [18]. The model is a box model of the climate where a similar atmospheric-cryospheric model as in the previous subsection is coupled to an ocean model with a sea-ice component. In [19], results of the model forced by constant annual mean insolation (no seasonal or Milankovitch forcing) are presented to assess the degree to which the internal processes (particularly sea-ice) may control glacial cycle variability. A typical result for (near) standard values of the parameters in [19] shows that oscillations with a time scale of about $100 \mathrm{kyr}$ are found. The proposed mechanism of the variability is referred to as the sea-ice switch, where rapid seaice growth and decay can act as a switch for the precipitation-temperature feedback affecting the growth and/or decay of ice sheets. The $100 \mathrm{kyr}$ time scale is due to the growth and decay of ice sheets which is coupled by relatively rapid sea ice changes. 
When this model is forced with Milankovitch insolation changes, nonlinear resonances may occur between the internal oscillation and the orbital forcing leading to time series which qualitatively resemble the observed records [20], which demonstrates how phase locking to Milankovitch forcing affects glacial cycles in this idealized model. These nonlinear resonances are likely to be present in every model where a strong nonlinear interaction is represented, explaining for example the good agreement between very conceptual models, where only a multiple state switch [7] is represented, and observations. In other words, even if the mechanism of the glacial-interglacial variability is incorrect, there may still be a good fit with the isotopic record. Due to the synchronization, a comparison between time series of simple models and isotope records is not mechanistically selective [21].

Although many bifurcation studies have been done on low-order models, there appear to have been no studies where numerical bifurcation analysis has been applied to spatially extended models of ice sheets. In this contribution, we make a first step in this direction, by looking for multiple equilibria and Hopf bifurcations in a two-dimensional model of a marine ice sheet.

\section{Methodology}

Consider in Fig. 4 a two-dimensional marine ice sheet situated on a bedrock topography in a Cartesian coordinate system. The ice sheet and bedrock are taken symmetrical, with a symmetry axis at $x=0$. The grounding line is indicated by $x_{g}$, the ice thickness by $h$, and the bedrock by $b$.

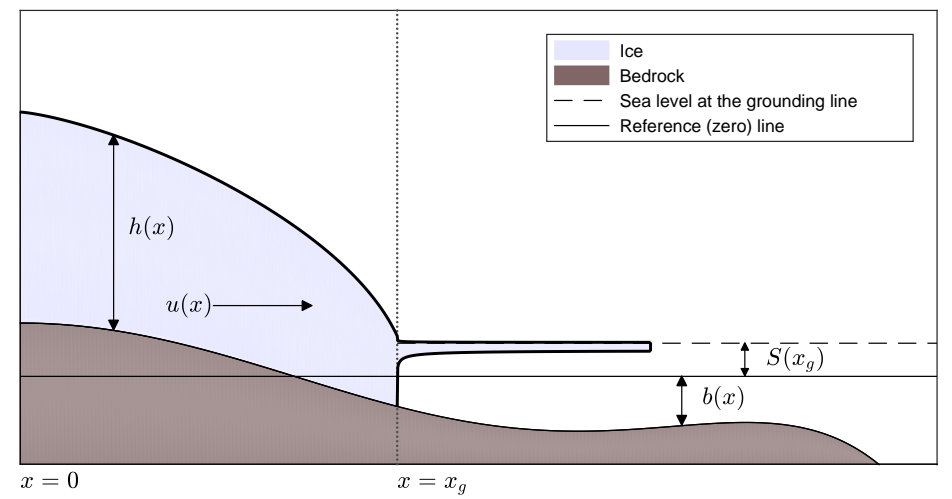

Fig. 4 A two-dimensional marine ice sheet. The ice thickness is given by $h(x)$ and the horizontal ice velocity by $u(x)$. Up until the grounding line $x_{g}$, the ice sheet rests on the bedrock $b(x)$. At $x_{g}$ the ice sheet extends into a floating ice shelf. 


\subsection{Model}

An introduction to ice-sheet and glacier modeling can be found in [22] and [23]. The dynamics of a marine ice sheet is modeled using the shallow-shelf approximation (SSA), which is obtained by simplifying the full Stokes problem for gravity driven ice flow [22]. The two-dimensional SSA, as implemented in [24], is used as a benchmark problem for the marine ice sheet intercomparison project (MISMIP, [25]). Since a thorough comparison with other results is available, this will be our model of choice for the bifurcation analysis. Conservation of mass gives:

$$
\frac{\partial h}{\partial t}+\frac{\partial(u h)}{\partial x}=a
$$

where $h$ is the ice thickness, $u$ the ice velocity and $a$ the accumulation rate. On a downward sloping bed (Fig. 4), the accumulation $a$ and the ice flux $u h$ at the grounding line are in equilibrium: a positive perturbation of the grounding line increases both the accumulation and the flux, leading to a zero net ice growth.

Conservation of momentum gives:

$$
\frac{\partial}{\partial x}\left[2 A^{-\frac{1}{n}} h\left|\frac{\partial u}{\partial x}\right|^{\frac{1}{n}-1} \frac{\partial u}{\partial x}\right]-C|u|^{(m-1)} u-\rho_{i} g h \frac{\partial(h-b)}{\partial x}=0
$$

where $A$ and $n$ are coefficients of Glen's flow law, a constitutive relation describing the rheology of ice ([23], [22], typically $n=3$ ). The ice density is given by $\rho_{i}, g$ is the gravitational acceleration and $b$ the bedrock taken positive in the downward direction. The consecutive terms in the momentum balance (3) represent longitudinal stresses, vertical shear stresses, and the driving stress respectively. The parameters $C$ and $m$ determine the sliding of the ice. Together, $A$ and $C$ effectively describe the size of the transition zone, i.e., the region in which the grounded sheet becomes afloat and transforms into an ice shelf.

The left boundary of the domain is located at an ice divide, a location in the ice with zero horizontal flow. Hence, we take $u=0$ at $x=0$. As we assume symmetry around $x=0$, we also require that

$$
\frac{\partial(h-b)}{\partial x}=0 \quad \text { for } x=0 .
$$

We will denote the grounding line by $x_{g}$. At $x_{g}$ the ice sheet becomes afloat and the following flotation condition holds:

$$
\rho_{i} h=\rho_{w} b \quad \text { for } x=x_{g}
$$

From an integration of the shelf flow [24], an extra condition at the grounding line is obtained: 


$$
2 A^{-\frac{1}{n}}\left|\frac{\partial u}{\partial x}\right|^{\frac{1}{n}-1} \frac{\partial u}{\partial x}=\frac{1}{2}\left(1-\frac{\rho_{i}}{\rho_{w}}\right) \rho_{i} g h \quad \text { for } x=x_{g} .
$$

Note that the profile of the shelf is not given by the SSA model, but obtained using an equilibrium analysis [23].

\subsection{Non-dimensional equations}

The first difficulty one encounters is the unknown right boundary of the problem given by the grounding line position $x_{g}$. As discussed in [24] and [26], a moving grid approach can be used to track $x_{g}$. Using a transformation $z=x / x_{g}$, the original domain $x \in\left[0, x_{g}\right]$ is mapped onto the fixed domain $z \in[0,1]$. As a result, the problem now has three unknowns: $h, u$ and $x_{g}$. The differential operators are transformed using the chain rule:

$$
\begin{aligned}
& \frac{\partial}{\partial t}=\frac{\partial}{\partial \tau} \frac{\partial \tau}{\partial t}+\frac{\partial}{\partial z} \frac{\partial z}{\partial t}=\frac{\partial}{\partial \tau}-\frac{z}{x_{g}} \frac{\mathrm{d} x_{g}}{\mathrm{~d} t} \frac{\partial}{\partial z} \\
& \frac{\partial}{\partial x}=\frac{\partial}{\partial \tau} \frac{\partial \tau}{\partial x}+\frac{\partial}{\partial z} \frac{\partial z}{\partial x}=\frac{1}{x_{g}} \frac{\partial}{\partial z}
\end{aligned}
$$

where $z$ and $\tau$ denote the independent variables in the transformed domain. Since we only transform in space we have that $\tau=t$. The transformations of (2)-(6) are then given by:

$$
\begin{gathered}
\frac{\partial h}{\partial \tau}-\frac{z}{x_{g}} \frac{\mathrm{d} x_{g}}{\mathrm{~d} \tau} \frac{\partial h}{\partial z}+\frac{1}{x_{g}} \frac{\partial(u h)}{\partial z}=a \\
\frac{1}{x_{g}^{\frac{1}{n}+1}} \frac{\partial}{\partial z}\left[2 A^{-\frac{1}{n}} h\left|\frac{\partial u}{\partial z}\right|^{\frac{1}{n}-1} \frac{\partial u}{\partial z}\right]-C|u|^{(m-1)} u-\frac{\rho_{i} g h}{x_{g}} \frac{\partial(h-b)}{\partial z}=0 \\
\frac{\partial(h-b)}{\partial z}=u=0 \quad \text { for } z=0 \\
\rho_{i} h=\rho_{w} b \quad \text { for } z=1, \\
2 A^{-\frac{1}{n}} \frac{1}{x_{g}^{\frac{1}{n}}}\left|\frac{\partial u}{\partial z}\right|^{\frac{1}{n}-1} \frac{\partial u}{\partial z}=\frac{1}{2}\left(1-\frac{\rho_{i}}{\rho_{w}}\right) \rho_{i} g h \quad \text { for } z=1 .
\end{gathered}
$$

To improve numerical accuracy the equations are non-dimensionalized. Let

$$
h=h_{0} \tilde{h}, \quad b=h_{0} \tilde{b}, \quad x_{g}=x_{0} \tilde{x}_{g}, \quad \tau=\tau_{0} \tilde{\tau}, \quad u=u_{0} \tilde{u}, \quad u_{0}=\frac{x_{0}}{\tau_{0}},
$$

with typical thickness $h_{0}=1 \times 10^{3} \mathrm{~m}$, horizontal extent $x_{0}=1 \times 10^{5} \mathrm{~m}$ and typical timescale $\tau_{0}=100 \mathrm{y}$. Substituting these expressions into (7) gives 


$$
\frac{h_{0}}{\tau_{0}}\left(\frac{\partial \tilde{h}}{\partial \tilde{\tau}}-\frac{z}{\tilde{x}_{g}} \frac{\mathrm{d} \tilde{x}_{g}}{\mathrm{~d} \tilde{\tau}} \frac{\partial \tilde{h}}{\partial z}+\frac{1}{\tilde{x}_{g}} \frac{\partial(\tilde{u} \tilde{h})}{\partial z}\right)=a \Leftrightarrow \frac{\partial \tilde{h}}{\partial \tilde{\tau}}-\frac{z}{\tilde{x}_{g}} \frac{\mathrm{d} \tilde{x}_{g}}{\mathrm{~d} \tilde{\tau}} \frac{\partial \tilde{h}}{\partial z}+\frac{1}{\tilde{x}_{g}} \frac{\partial(\tilde{u} \tilde{h})}{\partial z}=\Omega,
$$

where we let $\Omega=\frac{\tau_{0}}{h_{0}} a$. Similarly, the non-dimensionalized version of (8) is given by

$$
\frac{1}{\tilde{x}_{g}^{\frac{1}{n}+1}} \frac{\partial}{\partial z}\left[\tilde{h}\left|\frac{\partial \tilde{u}}{\partial z}\right|^{\frac{1}{n}-1} \frac{\partial \tilde{u}}{\partial z}\right]-\Gamma|\tilde{u}|^{(m-1)} \tilde{u}-\Lambda \frac{\rho_{i} g \tilde{h}}{\tilde{x}_{g}} \frac{\partial(\tilde{h}-\tilde{b})}{\partial z}=0
$$

where we introduce the new constants

$$
\Gamma:=C\left(\frac{x_{0}}{\tau_{0}}\right)^{m} /\left(2 A^{-\frac{1}{n}}\left(\frac{x_{0}}{\tau_{0}}\right)^{\frac{1}{n}} \frac{h_{0}}{x_{0}^{\frac{1}{n}+1}}\right) \quad \text { and } \quad \Lambda:=\frac{h_{0}^{2}}{x_{0}} /\left(2 A^{-\frac{1}{n}}\left(\frac{x_{0}}{\tau_{0}}\right)^{\frac{1}{n}} \frac{h_{0}}{x_{0}^{\frac{1}{n}+1}}\right) \text {. }
$$

Finally, at the boundaries we obtain

$$
\begin{gathered}
\frac{\partial(\tilde{h}-\tilde{b})}{\partial z}=\tilde{u}=0 \quad \text { for } z=0, \\
\rho_{i} \tilde{h}=\rho_{w} \tilde{b} \quad \text { for } z=1, \\
\frac{1}{\tilde{x}_{g}^{\frac{1}{n}}}\left|\frac{\partial \tilde{u}}{\partial z}\right|^{\frac{1}{n}-1} \frac{\partial \tilde{u}}{\partial z}=\Sigma\left(1-\frac{\rho_{i}}{\rho_{w}}\right) \rho_{i} g \tilde{h} \quad \text { for } z=1,
\end{gathered}
$$

with

$$
\Sigma:=\frac{1}{2} h_{0} /\left(2 A^{-\frac{1}{n}} \frac{1}{\tau_{0}^{\frac{1}{n}}}\right)
$$

\subsection{Numerical implementation}

From here on we omit the tildes and assume the unknowns are non-dimensional. As in [24], the domain $z \in[0,1]$ is discretized using a staggered grid with a fixed mesh-width: $\Delta z=1 /(N-1 / 2)$. The left boundary is taken at the vertex $i=1$ and the right boundary at the cell center $i=N+1 / 2$. Thus, for $i=1,2, \ldots, N$, we have vertices at $z_{i}=\Delta z(i-1)$ and cell centers at $z_{i+1 / 2}=\Delta z(i-1 / 2)$. The discretized solution values for ice thickness are located at the vertices $h_{i}$, while the values for ice velocity are positioned at the cell centers $u_{i+1 / 2}$.

The transformed and non-dimensionalized continuity equation (13) is discretized using a central difference for the stretching and an upwind discretization for the flux:

$\frac{\mathrm{d} h_{i}}{\mathrm{~d} \tau}-\frac{z_{i}}{x_{g}}\left(\frac{h_{i+1}-h_{i-1}}{2 \Delta z}\right) \frac{\mathrm{d} x_{g}}{\mathrm{~d} \tau}=-\frac{h_{i}\left(u_{i+1 / 2}+u_{i-1 / 2}\right)-h_{i-1}\left(u_{i-1 / 2}+u_{i-3 / 2}\right)}{2 x_{g} \Delta z}+\Omega_{i}$. 
At the left boundary symmetry requires

$$
\begin{aligned}
\left(h_{2}-h_{0}\right) /(2 \Delta z) & =0 \quad(\text { central }), \\
\left(h_{1}-h_{0}\right) / \Delta z & =0 \quad \text { (upwind) }, \\
u_{3 / 2}+u_{1 / 2} & =0, \quad u_{5 / 2}+u_{-1 / 2}=0 .
\end{aligned}
$$

Using these expressions we can resolve the dependence on nonexistent grid-points. For $i=1$ and $i=2$, mass conservation is therefore given by

$$
\begin{aligned}
& (i=1) \quad \frac{\mathrm{d} h_{1}}{\mathrm{~d} \tau}=-\frac{h_{1}\left(u_{3 / 2}+u_{5 / 2}\right)}{2 x_{g} \Delta z}+\Omega_{1}, \\
& (i=2) \quad \frac{\mathrm{d} h_{2}}{\mathrm{~d} \tau}-\frac{z_{2}}{x_{g}}\left(\frac{h_{3}-h_{1}}{2 \Delta z}\right) \frac{\mathrm{d} x_{g}}{\mathrm{~d} \tau}=-\frac{h_{2}\left(u_{5 / 2}+u_{3 / 2}\right)}{2 x_{g} \Delta z}+\Omega_{2} .
\end{aligned}
$$

Note that at the rightmost vertex $(i=N)$, the right hand side of the discretized mass conservation (20) does not contain any dependencies on nonexistent grid-points. In the left hand side we will need to use a one-sided difference for the stretching term.

Define $\Delta u_{i}:=u_{i+1 / 2}-u_{i-1 / 2}$. The momentum conservation (14) is discretized using central differences:

$$
\begin{aligned}
0= & \frac{1}{\left(x_{g} \Delta z\right)^{1+1 / n}}\left[h_{i+1}\left|\Delta u_{i+1}\right|^{1 / n-1} \Delta u_{i+1}-h_{i}\left|\Delta u_{i}\right|^{1 / n-1} \Delta u_{i}\right] \\
& -\Gamma\left|u_{i+1 / 2}\right|^{m-1} u_{i+1 / 2}-\Lambda\left(\frac{h_{i}+h_{i+1}}{2}\right) \frac{\rho_{i} g}{x_{g} \Delta z}\left[h_{i+1}-b_{i+1}-h_{i}+b_{i}\right] .
\end{aligned}
$$

At the left boundary we let $\Delta u_{1}=2 u_{3 / 2}$. At the right boundary we impose the following discretization of (18) with a substituted flotation condition $\rho_{i} h_{N}=\rho_{w} b_{N}$ (cf. (17)):

$$
0=\frac{1}{\left(x_{g} \Delta z\right)^{1 / n}}\left|\Delta u_{N}\right|^{1 / n-1}\left(\Delta u_{N}\right)-\Sigma\left(1-\frac{\rho_{i}}{\rho_{w}}\right) \rho_{w} g b_{N} .
$$

The discretization contains $N$ unknown $h_{i}, N$ unknown $u_{i+1 / 2}$ and an unknown grounding line position $x_{g}$. To achieve a closed system of $2 N+1$ equations, the flotation criterion at the cell center $z_{N+1 / 2}$ is prescribed using an extrapolation of the thickness, which gives the closing requirement:

$$
0=3 h_{N}-h_{N-1}-2 \frac{\rho_{w}}{\rho_{i}} b_{N} .
$$

Finally we obtain a problem of the form

$$
M \frac{\mathrm{d}}{\mathrm{d} t} \mathbf{x}=F(\mathbf{x}, \lambda), \text { with } \mathbf{x}=\left[\begin{array}{c}
\mathbf{h} \\
\mathbf{u} \\
x_{g}
\end{array}\right] .
$$


The unknown functions are discretized: $\mathbf{h}, \mathbf{u} \in \mathbb{R}^{N}$. The real-valued matrix $M \in$ $\mathbb{R}^{(2 N+1) \times(2 N+1)}$ and nonlinear operator $F: \mathbb{R}^{2 N+1} \rightarrow \mathbb{R}^{2 N+1}$ are given by

$$
M=\left[\begin{array}{ccc}
I & 0 & M_{\mathrm{str}}\left(\mathbf{h}, x_{g}\right) \\
0 & 0 & 0 \\
0 & 0 & 0
\end{array}\right], \quad F=\left[\begin{array}{c}
F_{\mathrm{mass}}\left(\mathbf{h}, \mathbf{u}, x_{g}\right) \\
F_{\mathrm{mom}}\left(\mathbf{h}, \mathbf{u}, x_{g}\right) \\
F_{\mathrm{flot}}\left(\mathbf{h}, x_{g}\right)
\end{array}\right]
$$

Here, $M_{\text {str }}\left(\mathbf{h}, x_{g}\right) \in \mathbb{R}^{N}$ is the discretization of the stretching in the left-hand side of Equations 201-22). $F_{\text {mass }}\left(\mathbf{h}, \mathbf{u}, x_{g}\right) \in \mathbb{R}^{N}$ is given by the right-hand side of discretizations 201-22). Similarly, $F_{\text {mom }}\left(\mathbf{h}, \mathbf{u}, x_{g}\right) \in \mathbb{R}^{N}$ and $F_{\text {flot }}\left(\mathbf{h}, \mathbf{u}, x_{g}\right) \in \mathbb{R}$ are given by the right hand sides of the discretized momentum equation and flotation criterion (23)-(24).

\subsection{Pseudo-arclength continuation}

The discretized equations give a problem of the form

$$
M \frac{\mathrm{d} \mathbf{x}}{\mathrm{d} t}=F(\mathbf{x}, \lambda)
$$

where $M$ and $F(\cdot)$ are linear and non-linear operators respectively. We explicitly introduce the parameter dependence $\lambda$ since we are interested in solution branches $(\mathbf{x}, \lambda)$ satisfying $F(\mathbf{x}, \lambda)=0$. For example, our first parameter of interest will be the temperature, which is present in the coefficient $A$ in Glen's flow law.

Various continuation techniques exist to trace a stationary solution branch while varying a parameter. A successful approach is to parameterize a solution branch with a pseudo-arclength parameter $s: \gamma(s)=(\mathbf{x}(s), \lambda(s))$ and impose an approximate normalization condition on the tangent, to close the system of equations: $\dot{\mathbf{x}}^{T}(\mathbf{x}-$ $\left.\mathbf{x}_{0}\right)+\dot{\lambda}\left(\lambda-\lambda_{0}\right)-\Delta s=0$, where $\left(\mathbf{x}_{0}, \lambda_{0}\right)$ is an initial known stationary solution, $(\dot{\mathbf{x}}, \dot{\lambda})$ the tangents w.r.t. the arclength parameter at $\left(\mathbf{x}_{0}, \lambda_{0}\right)$ and $\Delta s$ a specified step size [27, 28].

To find a new point on the solution branch a predictor-corrector method is used. A suitable tangent predictor is given by

$$
\begin{gathered}
\mathbf{x}^{1}=\mathbf{x}_{0}+\Delta s \dot{\mathbf{x}}, \\
\lambda^{1}=\lambda_{0}+\Delta s \dot{\lambda} .
\end{gathered}
$$

Note that the prediction is denoted by $\left(\mathbf{x}^{1}, \lambda^{1}\right)$, whereas an actual new solution will be denoted by $\left(\mathbf{x}_{1}, \lambda_{1}\right)$. The correction onto the solution branch is made through the solution of the nonlinear system given by

$$
\begin{aligned}
F(\mathbf{x}, \lambda) & =0, \\
\dot{\mathbf{x}}^{T}\left(\mathbf{x}-\mathbf{x}_{0}\right)+\dot{\lambda}\left(\lambda-\lambda_{0}\right)-\Delta s & =0 .
\end{aligned}
$$


A Newton-Raphson root finding procedure, initialized with the prediction $\left(\mathbf{x}^{1}, \lambda^{1}\right)$, gives the following iteration:

$$
\left[\begin{array}{cc}
F_{\mathbf{x}} & F_{\lambda} \\
\dot{\mathbf{x}}^{T} & \dot{\lambda}
\end{array}\right]\left[\begin{array}{c}
\Delta \mathbf{x} \\
\Delta \lambda
\end{array}\right]=\left[\begin{array}{c}
-F\left(\mathbf{x}^{k}, \lambda\right) \\
\Delta s-\dot{\mathbf{x}}^{T}\left(\mathbf{x}^{k}-\mathbf{x}_{0}\right)-\dot{\lambda}\left(\lambda^{k}-\lambda_{0}\right)
\end{array}\right]
$$

where $\Delta \mathbf{x}:=\mathbf{x}^{k+1}-\mathbf{x}^{k}, \Delta \lambda:=\lambda^{k+1}-\lambda^{k}$ and $\left[F_{\mathbf{x}}, F_{\lambda}\right]$ is the Jacobian matrix of $F$. If this iteration converges a new stationary solution $\left(\mathbf{x}_{1}, \lambda_{1}\right)$ has been found. At a fold bifurcation the Jacobian matrix $F_{\mathbf{x}}$ will have a zero eigenvalue, yet the system in (31) remains non-singular, and the continuation is able to trace the solution branch into its unstable domain.

When a stationary solution $\overline{\mathbf{x}}$, satisfying

$$
F(\overline{\mathbf{x}}, \lambda)=0,
$$

has been found, its stability can be investigated with a perturbation $\overline{\mathbf{x}}+\tilde{\mathbf{x}}$ and a Taylor expansion around the stationary solution:

$$
\begin{gathered}
M \frac{\mathrm{d}}{\mathrm{d} t}(\overline{\mathbf{x}}+\tilde{\mathbf{x}})=M \frac{\mathrm{d}}{\mathrm{d} t} \overline{\mathbf{x}}+M \frac{\mathrm{d}}{\mathrm{d} t} \tilde{\mathbf{x}}=F(\overline{\mathbf{x}}+\tilde{\mathbf{x}}, \lambda) \approx F(\overline{\mathbf{x}}, \lambda)+F_{\overline{\mathbf{x}}}(\overline{\mathbf{x}}, \lambda) \tilde{\mathbf{x}} \Leftrightarrow \\
M \frac{\mathrm{d}}{\mathrm{d} t} \tilde{\mathbf{x}}=F_{\overline{\mathbf{x}}}(\overline{\mathbf{x}}, \lambda) \tilde{\mathbf{x}} .
\end{gathered}
$$

Solutions of (33) are of the form $\tilde{\mathbf{x}}=\hat{\mathbf{x}} e^{\sigma t}$. Substitution gives a generalized eigenvalue problem:

$$
\sigma M \hat{\mathbf{x}}=F_{\overline{\mathbf{x}}}(\overline{\mathbf{x}}, \lambda) \hat{\mathbf{x}} .
$$

The stability of a stationary solution depends on the sign of the real part of the eigenvalues. If we find an eigenvalue with positive real part the solution contains a growing mode and is thus unstable.

\section{Results}

\subsection{Bifurcation diagram}

First we consider the case of constant accumulation, represented by a constant $a$, together with a fixed bedrock $b(x)$. The bedrock profile is chosen as in [24]:

$$
b(x)=-\left(729-2184.8\left(\frac{x}{S}\right)^{2}+1031.72\left(\frac{x}{S}\right)^{4}-151.72\left(\frac{x}{S}\right)^{6}\right)
$$

with $S=750 \times 10^{3} \mathrm{~m}$. To find a stationary solution, an ice sheet surface profile of the form 

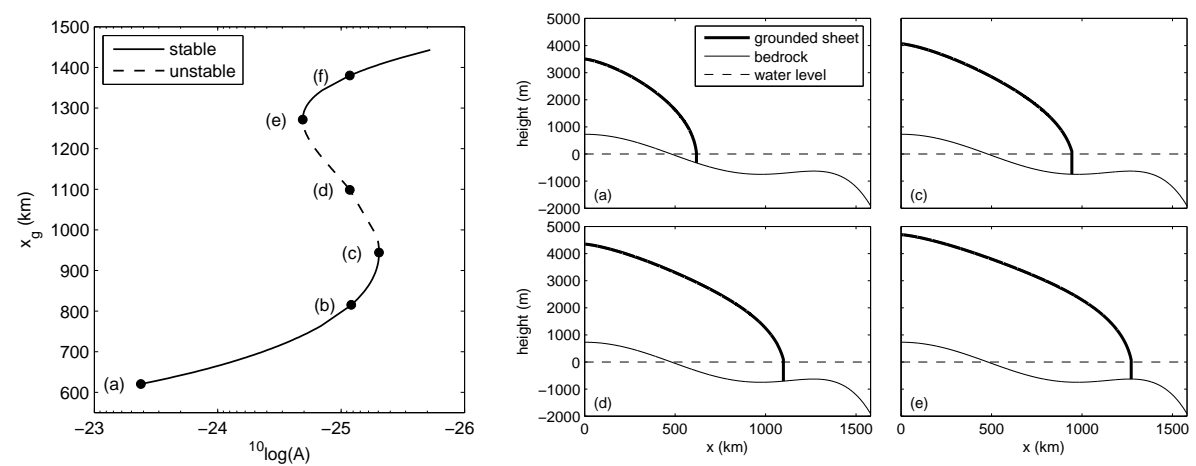

Fig. 5 One-parameter bifurcation diagram (left) and solutions (right), decreasing the parameter $A$ in the SSA model which corresponds to a decrease in temperature and an increase in ice growth. The bedrock contains an upward slope which admits multiple steady states ((b),(d) and (f)) for a constant parameter. Eigenvalue analysis shows two saddle-node bifurcations: a single eigenvalue crosses the imaginary axis to the positive right half plane at (c) and returns to the left half plane at (e). The number of grid-points is $N=1600$; the other parameters are given in Table 1

$$
s=h-b=C_{1} \sqrt{1-\left(\frac{x}{x_{g}}\right)^{2}}+C_{2}, \text { with } x \in\left[0, x_{g}\right],
$$

is used as initial guess for the Newton-Raphson iteration solving $F(\mathbf{x}, \lambda)=0$. Typically, $C_{1}=3 \times 10^{3}$ and $C_{2}$ is chosen such that the flotation criterion is satisfied: $C_{2}=\left(\rho_{w} / \rho_{i}-1\right) b_{g}$, where $b_{g}$ is the bedrock at the grounding line. The analytic profile contains a steep gradient at the grounding line, which is essential for a good convergence of the root finding process.

After an initial equilibrium profile is obtained for $A=4.6416 \times 10^{-24}$, a pseudoarclength continuation traces the solution branch $\gamma(s)$ in the direction of decreasing $A$, see Figure 5 . The presented bifurcation diagram confirms the multiple equilibria regime associated with the hysteretic behavior shown in [24]. For a fixed value of the parameter $A$, three equilibria are distinguished and marked as (b), (d) and (f). At the point (c) a saddle-node bifurcation occurs and an eigenvalue is observed to cross the imaginary axis to the right half plane. At (e), the same eigenvalue returns to the right half plane through a second saddle-node bifurcation. The values of the parameters are summarized in Table 1.

\subsection{Numerical accuracy}

In order to investigate the numerical accuracy of the discretization and continuation methodology we perform a convergence experiment, see Table 2 Let the error in the approximated bifurcation point $A_{N}$ be proportional to a power of the mesh-width: 


\begin{tabular}{|c|c|}
\hline$C$ & $7.624 \times 10^{6} \mathrm{~Pa} \mathrm{~m}^{-1 / 3} \mathrm{~s}^{1 / 3}$ \\
$m$ & $1 / 3$ \\
$n$ & 3 \\
$g$ & $9.8 \mathrm{~m} \mathrm{~s}^{-2}$ \\
$\rho_{i}$ & $900 \mathrm{~kg} \mathrm{~m}^{-3}$ \\
$\rho_{w}$ & $1000 \mathrm{~kg} \mathrm{~m}^{-3}$ \\
$a$ & $0.3 \mathrm{~m} \mathrm{y}^{-1}$ \\
\hline
\end{tabular}

\begin{tabular}{|c|c|}
\hline & $A\left(\mathrm{~s}^{-1} \mathrm{~Pa}^{-3}\right)$ \\
\hline (a) & $4.6416 \times 10^{-24}$ \\
(b) & $8.5014 \times 10^{-26}$ \\
(c) & $4.9274 \times 10^{-26}$ \\
(d) & $8.5014 \times 10^{-26}$ \\
(e) & $2.0450 \times 10^{-25}$ \\
(f) & $8.5014 \times 10^{-26}$ \\
\hline
\end{tabular}

Table 1 Parameter values for the experiment in Figure 5 similar to the values chosen in [24] and [25].

$$
A_{N}=A+\alpha(\Delta z)^{\beta}
$$

where $A$ denotes the actual bifurcation point and $\alpha$ and $\beta$ are constants. We define a difference between subsequent mesh-halvings $D_{N}:=A_{N}-A_{N / 2}$ and let $\Delta z \approx 1 / N$. Then, the ratio between consecutive differences only depends on the power $\beta$ :

$$
R_{N}:=\frac{D_{N / 2}}{D_{N}}=\frac{A_{N / 2}-A_{N / 4}}{A_{N}-A_{N / 2}}=\frac{(1 / 2)^{\beta}-1}{(1 / 4)^{\beta}-(1 / 2)^{\beta}} .
$$

\begin{tabular}{|c|c|c|c|}
\hline$N$ & $A_{N}$ & $D_{N}$ & $R_{N}$ \\
\hline 50 & $2.61941 \times 10^{-26}$ & & \\
100 & $3.48732 \times 10^{-26}$ & $8.67909 \times 10^{-27}$ & \\
200 & $4.14983 \times 10^{-26}$ & $6.62512 \times 10^{-27}$ & 1.31003 \\
400 & $4.56744 \times 10^{-26}$ & $4.17606 \times 10^{-27}$ & 1.58645 \\
800 & $4.80250 \times 10^{-26}$ & $2.35065 \times 10^{-27}$ & 1.77655 \\
1600 & $4.92741 \times 10^{-26}$ & $1.24911 \times 10^{-27}$ & 1.88186 \\
\hline
\end{tabular}

Table 2 Convergence of the first bifurcation (point (c) in Figure 5 .

From Table 2 we suspect $R_{N} \rightarrow 2$ as $N$ becomes large, corresponding to $\beta=1$. The scheme must therefore be of first-order accuracy, which is undoubtedly due to the first-order upwind discretization in the continuity equation (20). Unfortunately, the upwind discretization of the ice flux is essential to the stability of the scheme. We conclude that a higher order upwind scheme should be considered in 20).

\subsection{Mechanism}

The advantage of the approach chosen here is that the spatial patterns of perturbations destabilizing the marine ice sheet can be determined from the eigenvectors in (34). For the unstable equilibrium (d) in Figure 5 it is of interest to examine the eigenmode with a positive growth factor, showing in detail the characteristics of the instability. The eigenvector is made available using (34) and is depicted for the 

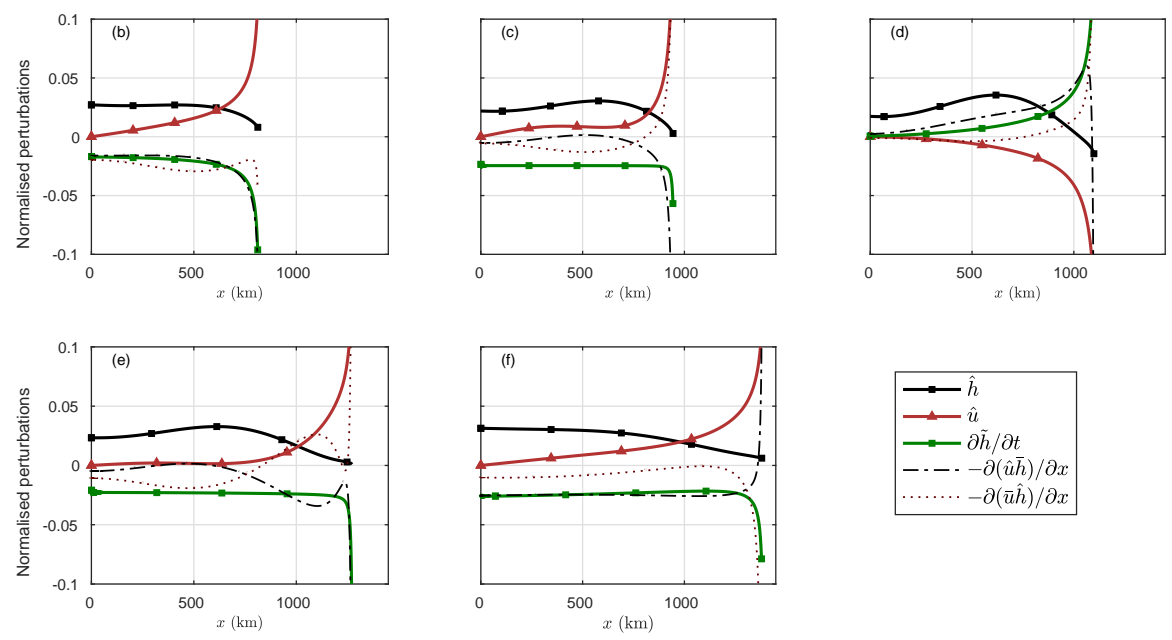

Fig. 6 Normalized components of the eigenmode that becomes unstable. The corresponding steady states are located at the points (b)-(f), described in Figure 5 and Table 1 We distinguish between a perturbation pattern related to sheet thickness $\hat{h}$ and a pattern related to ice velocity $\hat{u}$. The signs of the eigenvectors are taken such that the perturbation in the grounding line is positive. From the eigenvectors and the equilibrium solution we compute a normalized spatial pattern of the evolution $\partial \tilde{h} / \partial t$, together with normalized components $-\partial(\hat{u} \bar{h}) / \partial x$ and $-\partial(\bar{u} \hat{h}) / \partial x$.

steady states (b),(c),(d),(e) and (f) in Figure 6 The perturbations in thickness and velocity are taken corresponding to a positive grounding line perturbation. Note that a perturbation of the solution vector has the form $\hat{\mathbf{x}} e^{\sigma t}=\left[\hat{\mathbf{h}}, \hat{\mathbf{u}}, \hat{x}_{g}\right]^{T} e^{\sigma t}$, with $\hat{x}_{g}$ the scalar grounding line perturbation. An eigenvector with corresponding eigenvalue $\sigma>0$ and $\hat{x}_{g}<0$ gives the destabilizing pattern for unstable ice sheet retreat. By adjusting the sign of the eigenvector, such that $\hat{x}_{g}>0$, we restrict our exposition to destabilizing patterns for unstable ice sheet growth.

At the grounding line $x_{g}$, the perturbation of the unstable steady state (d) shows a slight decrease in ice thickness, while at (b),(c),(e) and (f) a slight increase is observed. In the interior of the ice sheet a relatively large increase in ice thickness is visible for the unstable equilibrium (d), indicating interior ice growth due to an imbalance between global accumulation and ice flux at the grounding line.

The velocity perturbation at the grounding line shows an increase for stable states and a clear decrease for the unstable state (d). Together with the negative perturbation in thickness this implies that, at (d), there must be a decrease in flux $u h$ across the grounding line for a positive perturbation $\hat{x}_{g}>0$. An increase in grounding line position implies a rise in global accumulation, hence the reduction in grounding line flux implies a net ice growth, confirming the marine ice sheet instability hypothesis. Note that a similar result holds if we take the perturbation in $x_{g}$ negative, giving a net ice loss and a retreat from equilibrium.

The continuation approach allows an efficient computation of flux perturbations using (34). From a linear stability analysis of (2) with perturbation $\tilde{h}=c \hat{h}, \tilde{u}=$ 
$c \hat{u}$ around an equilibrium $\bar{h}, \bar{u}$ we obtain an evolution equation for the thickness perturbation $\tilde{h}$ :

$$
\begin{aligned}
& \frac{\partial \tilde{h}}{\partial t}+c \frac{\partial \hat{q}}{\partial x}=0, \quad \text { with } c>0, \\
& \hat{q}(x)=\hat{u} \bar{h}+\bar{u} \hat{h},
\end{aligned}
$$

where we neglect higher order terms. At the unstable steady state (d) in Figure 6 , the thickness perturbation (green squares) shows positive growth, whereas the other points show a dampening. These patterns are determined by spatial derivatives of the perturbed advection of the steady thickness $\hat{u} \bar{h}$ (black dash-dotted line)and the advected thickness perturbation $\bar{u} \hat{h}$ (red dotted line). The latter clearly dominates the instability in (d). Note that at the bifurcation points (c) and (e) the components of the perturbation flux have a compensating effect.

To investigate how a perturbation changes from stable to unstable through the saddle-node bifurcation $L_{1}$, we compute the accumulation and grounding line fluxes. The steady state $(\bar{h}, \bar{u})$ gives a balance:

$$
\bar{q}(x)=\bar{u} \bar{h}=a x .
$$

In Figure 7 we show perturbations of the balance 410 at the grounding line. A
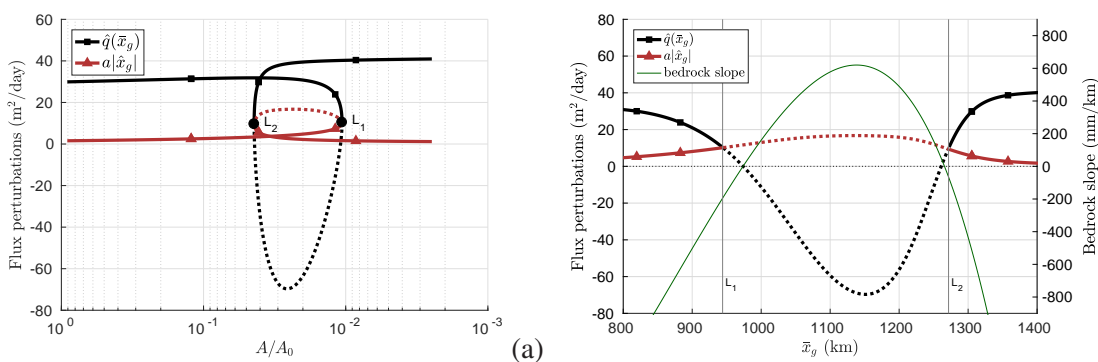

(b)

Fig. 7 Perturbations in grounding line and accumulation flux against the parameter $A$ (a) and as a function of $x_{g}(\mathrm{~b})$, together with the bedrock slope. The first and second saddle-node bifurcations are marked $L_{1}$ and $L_{2}$. Dashed lines correspond to growing perturbations of unstable steady states. The perturbations $\hat{q}\left(\bar{x}_{g}\right)$ and $a\left|\hat{x}_{g}\right|$ correspond to a positive grounding line perturbation $\hat{x}_{g}>0$. At $L_{1}$ and $L_{2}$, the grounding line bedrock slope is $-195 \mathrm{~mm} / \mathrm{km}$ and $-65 \mathrm{~mm} / \mathrm{km}$ respectively. The maximum slope in the unstable regime is $620 \mathrm{~mm} / \mathrm{km}$. Note that grounding line flux perturbations depend on the steady grounding line position $\bar{x}_{g}$, whereas accumulation flux perturbations depend on the grounding line perturbation $\hat{x}_{g}$.

perturbation in accumulation flux is given by $a\left|\hat{x}_{g}\right|$, a grounding line flux perturbation by $\hat{q}\left(\bar{x}_{g}\right)$ in 40 . The perturbations are plotted against $A / A_{0}$ (Figure $7 \mathrm{~h}$ ) and $\bar{x}_{g}$ (Figure $7 \mathrm{~b}$ ), together with the bifurcation points and the bedrock slope. At the first saddle-node bifurcation $L_{1}$, the flux $\hat{q}\left(x_{g}\right)$ becomes smaller than the accumulation $a\left|\hat{x}_{g}\right|$. Beyond this point, a change in accumulation due to $\hat{x}_{g}$ is not balanced by the 
grounding line flux and, hence, the perturbation $\tilde{\mathbf{x}}$ changes from damped to growing. At $L_{2}, \hat{q}\left(x_{g}\right)$ becomes greater than $a\left|\hat{x}_{g}\right|$ and the mode is damped again.

In Figure $7 \mathrm{~b}$ we also plot the bedrock slope, taken positive when the bed elevation increases with $x$, that is

$$
r_{\text {bed }}=-b^{\prime}\left(x_{g}\right)
$$

with $b(x)$ as in (35). Note that the sign switch in $\hat{q}\left(x_{g}\right)$ coincides with the sign switch in the bedrock slope. The grounding line flux will increase for a positive $\hat{x}_{g}$ between the bifurcation $L_{1}$ and the point of zero bedrock slope, but, since the change is less than the change in accumulation, the ice sheet will grow. Thus, Figure 7 confirms that an eigenvalue of the 'full model' in [24] becomes positive when $a\left|\hat{x}_{g}\right|-\hat{q}\left(x_{g}\right)>$ 0 .

Using a continuation of steady states with the original SSA equations, we find that the flux perturbations and their relative magnitude fully describe the instability mechanism, confirming the analysis in [29]. In addition, the eigenvectors reveal destabilizing interior patterns with, most interestingly, interior thickness anomalies and their advection. These turn out to play a major role in the unstable growth and retreat of the ice sheet.

\subsection{Glacial isostatic adjustment}

The simplest model describing the interaction between an ice sheet and the underlying bedrock is a local lithosphere, relaxing asthenosphere (LLRA) model. An equilibrium argument [22] gives:

$$
\rho_{a} g\left(b^{*}-b^{0}\right)=\rho_{i} g h \Rightarrow\left(b^{*}-b^{0}\right)=\frac{\rho_{i}}{\rho_{a}} h,
$$

with $\rho_{a}$ the density of the asthenosphere, $b^{*}$ the equilibrium bedrock and $b^{0}$ the initial, load-free bedrock. Due to the highly viscous asthenosphere the equilibrium bedrock is reached after a significant response time $\tau_{a}$. The evolution of the bedrock can be modeled using

$$
\frac{\partial b}{\partial t}=\frac{1}{\tau_{a}}\left[\left(b^{*}-b^{0}\right)-\left(b-b^{0}\right)\right]=\frac{1}{\tau_{a}}\left[\frac{\rho_{i}}{\rho_{a}} h-\left(b-b^{0}\right)\right],
$$

with $b$ the actual bedrock profile.

Adding this elastic bedrock to the SSA model means introducing a new unknown and a new discretized differential equation. To remain consistent with the implementation in Section 3.3 we need to perform the transformation $z=x / x_{g}$, giving

$$
\frac{\partial b}{\partial \tau}-\frac{z}{x_{g}} \frac{\mathrm{d} x_{g}}{\mathrm{~d} \tau} \frac{\partial b}{\partial z}=\frac{1}{\tau_{a}}\left[\frac{\rho_{i}}{\rho_{a}} h-\left(b-b^{0}\right)\right] .
$$


Non-dimensionalizing (45) is straightforward. Using a central difference for the stretching we obtain the following discretization:

$$
\frac{\mathrm{d} b_{i}}{\mathrm{~d} \tau}-\frac{z}{x_{g}} \frac{\mathrm{d} x_{g}}{\mathrm{~d} \tau}\left(\frac{b_{i+1}-b_{i-1}}{2 \Delta z}\right)=\frac{1}{\tau_{a}}\left[\frac{\rho_{i}}{\rho_{a}} h_{i}-\left(b_{i}-b_{i}^{0}\right)\right] .
$$

Symmetry at the left boundary gives a vanishing spatial derivative. At the grounding line the pressure exerted by a column of ice equals that of the column of water: $\rho_{i} h_{N}=\rho_{w} b_{N}$. Substituting the flotation criterion gives the following discretization for the right boundary of the bedrock equation:

$$
\frac{\mathrm{d} b_{N}}{\mathrm{~d} \tau}-\frac{z}{x_{g}} \frac{\mathrm{d} x_{g}}{\mathrm{~d} \tau}\left(\frac{\frac{\rho_{i}}{\rho_{w}} h_{N}-b_{N-1}}{\Delta z}\right)=\frac{\rho_{w}-\rho_{a}}{\rho_{a} \tau_{a}}\left[b_{N}-\frac{\rho_{a}}{\rho_{a}-\rho_{w}} b_{N}^{0}\right] .
$$

Equation (47) acts as a Dirichlet boundary condition in the stationary case. Note that, with this boundary, we assume the bedrock is adjusted regardless of the type of material that is on top of it. Hence, the initial load-free bedrock $b^{0}$ cannot be subject to a water load. At the grounding line, ice and water exert the same pressure such that the bedrock extends continuously into its submerged part.

Again we let the problem have the form

$$
M \frac{\mathrm{d}}{\mathrm{d} t} \mathbf{x}=F(\mathbf{x}, \lambda)
$$

but now with $\mathbf{x}=\left[\mathbf{h}^{T}, \mathbf{u}^{T}, \mathbf{b}^{T}, x_{g}\right]^{T}, \mathbf{h}, \mathbf{u}, \mathbf{b} \in \mathbb{R}^{N}, M \in \mathbb{R}^{(3 N+1) \times(3 N+1)}$ and $F$ : $\mathbb{R}^{(3 N+1)} \rightarrow \mathbb{R}^{(3 N+1)}$, given by

$$
M=\left[\begin{array}{cccc}
I & 0 & 0 & M_{\text {mass }}\left(\mathbf{h}, x_{g}\right) \\
0 & 0 & 0 & 0 \\
0 & 0 & I & M_{\text {bed }}\left(\mathbf{b}, x_{g}\right) \\
0 & 0 & 0 & 0
\end{array}\right], \quad F=\left[\begin{array}{c}
F_{\text {mass }}\left(\mathbf{h}, \mathbf{u}, \mathbf{b}, x_{g}\right) \\
F_{\text {mom }}\left(\mathbf{h}, \mathbf{u}, \mathbf{b}, x_{g}\right) \\
F_{\text {bed }}\left(\mathbf{h}, \mathbf{b}, x_{g}\right) \\
F_{\text {flot }}\left(\mathbf{h}, \mathbf{b}, x_{g}\right)
\end{array}\right] .
$$

The functions $M_{\text {mass }}$ and $M_{\text {bed }}$ give the discretizations of the stretchings in the left hand side of the continuity and bedrock equation, $F_{\mathrm{bed}} \in \mathbb{R}^{N}$ gives the discretization of the elastic bedrock equation (46). The other functions are the same as in Section 3.3. except with their dependence on $\mathbf{b}$ made explicit.

Instead of having a constant accumulation $a$, as in [30] we will give it a linear dependence on the surface height $s(x)=h(x)-b(x)$ and a ceiling $a_{\max }$ :

$$
a(s)=\min \left(a_{\max }, \theta(s-E)\right),
$$

where $\theta$ is an accumulation gradient and $E$ the equilibrium height. Below $E$ we have mass loss, i.e. ablation, the opposite of accumulation. The balance between accumulation and ablation is governed by air temperature, which we assume to decrease with increasing surface height. 
The implementation of the height-dependent accumulation is straightforward, mainly requiring a few extra dependencies on $h$ and $b$ in the Jacobian matrix. To obtain a smooth transition from the linear function $\theta(s-E)$ to the ceiling $a_{\max }$, we use an approximation to the Heavyside function:

$$
a(s)=a_{\max }-\frac{1}{2}\left(1+\tanh \left(\frac{a_{\max }-\theta(s-E)}{\varepsilon}\right)\right)\left(a_{\max }-\theta(s-E)\right),
$$

with $\varepsilon$ moderately small.

Recall the bedrock $b$ as given in 35 . Now that we have implemented the isostatic adjustment, we need to define a smooth original bedrock $b^{0}$ that is not subject to a water load. Eventually, the bedrock will only be partially submerged, but adjusting $b$ only within the submerged sub-interval introduces unfavorable discontinuities. For that reason we will obtain $b^{0}$ using a global adjustment.

Consider the stationary case of (44). Replacing the ice load $\rho_{i} h$ with a water load $\rho_{w} b$ gives the required adjustment:

$$
b^{0}=\frac{\rho_{a}-\rho_{w}}{\rho_{a}} b .
$$

The added components open up a multitude of possible continuation parameters. Here we will restrict ourselves to a continuation in the equilibrium height $E$, to pursue oscillatory solutions due to the load accumulation feedback [31]: as ice thickness increases due to accumulation, the surface height may decrease as a result of isostatic adjustment (with a delay $\tau_{a}$ ), leading to a decrease in accumulation. This feedback suggests the possibility of oscillatory solutions within a certain parameter regime.

A Hopf bifurcation occurs when a steady periodical solution emerges from a fixed point. In the spectrum given by the eigenvalue analysis, a Hopf bifurcation corresponds to a complex conjugate pair crossing the imaginary axis from the left to the right half plane. The result of a continuation in $E$ is shown in Figure 8 An additional list of parameters is given in Table 3 .

\begin{tabular}{|r|l|l|}
\hline$\tau_{a}$ & $10000 \mathrm{y}$ & asthenosphere relaxation timescale \\
$\rho_{a}$ & $3300 \mathrm{~kg} \mathrm{~m}^{-3}$ & asthenosphere density \\
$a_{\mathrm{max}}$ & $0.1 \mathrm{~m} \mathrm{y}^{-1}$ & maximum accumulation \\
$\theta$ & $0.001 \mathrm{y}^{-1}$ & accumulation gradient \\
$A$ & $1.8969 \times 10^{-26} \mathrm{~s}^{-1} \mathrm{~Pa}^{-3}$ & Glen's flow law rheology parameter \\
\hline
\end{tabular}

Table 3 Parameter values for the experiment in Figure 8 The accumulation ceiling and gradient are chosen similar to [30]. Parameters not mentioned here remain equal to the ones given in Table 1

A Hopf bifurcation is detected at $E=1.04 \times 10^{3} \mathrm{~m}$, with complex conjugate eigenpair $\sigma=\sigma_{R} \pm i \sigma_{I} \approx 0 \pm i 0.006$. The corresponding complex eigenmode $\hat{x}=$ $\hat{x}_{R} \pm i \hat{x}_{I}$ describes the perturbation destabilizing the solution. The real part of the 

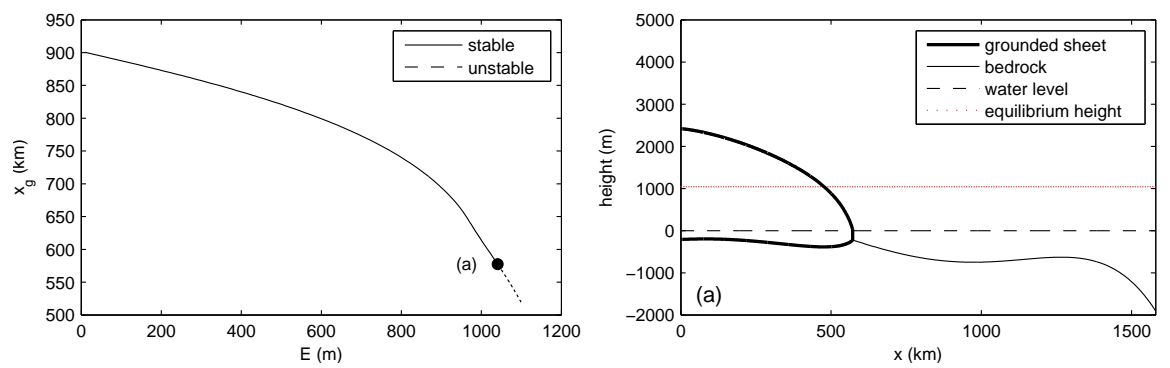

Fig. 8 Bifurcation diagram (left) and solution (right) of a continuation in equilibrium height $E$, starting at $E=0$. A Hopf bifurcation is detected at (a): $E=1.04 \times 10^{3} \mathrm{~m}$ and the corresponding solution is plotted on the right. Instances of the oscillatory perturbation corresponding to the complex conjugate eigenpair are plotted in Figure 9 The number of grid-points is $N=800$, the other parameters are given in Table 3
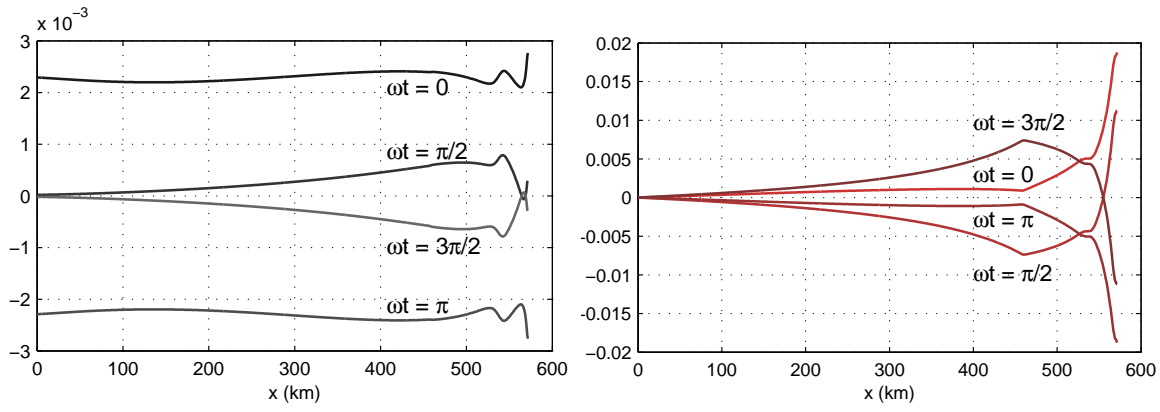

Fig. 9 Oscillating perturbation in ice thickness (left) and velocity (right), given by the eigenvector corresponding to the complex conjugate eigenpair at the Hopf bifurcation $E=1.04 \times 10^{3} \mathrm{~m}$. Several instances of the perturbation are obtained using [53). The angular frequency is given by $\omega=\sigma_{I}$.

perturbation gives a disturbance profile [27]:

$$
\operatorname{Re}\left(\hat{\mathbf{x}} e^{\sigma t}\right)=e^{\sigma_{R} t}\left[\hat{x}_{R} \cos \left(\sigma_{I} t\right)-\hat{x}_{I} \sin \left(\sigma_{I} t\right)\right] .
$$

At the bifurcation we have $\sigma_{R}=0$, then $\hat{x}_{R}$ and $\hat{x}_{I}$ give two instances of the oscillatory perturbation: $\hat{x}_{R}$ at $\sigma_{I} t=0$ and $\hat{x}_{I}$ at $\sigma_{I} t=3 \pi / 2$, see Figure 9 The nondimensional period is given by $\hat{T}=\frac{2 \pi}{\sigma_{I}} \approx 1.047 \times 10^{3}$. As the timescale in the experiment is taken $\tau_{0}=100 \mathrm{y}$, the dimensional period is $T=1.047 \times 10^{5} \mathrm{y}$.

The obtained oscillating perturbation demonstrates the dynamics of the load accumulation feedback. For the ice thickness, the perturbation in the interior seems to be constantly ahead of the perturbation at the grounding line. Note that the region between the ice divide and the point at which the surface attains the equilibrium height $(x \approx 476 \mathrm{~km})$ is subject to a net accumulation. From the perturbation in thickness we see that the feedback is clearly driven by the accumulation. 
Beyond $x \approx 476 \mathrm{~km}$ there is a net ablation. In this region the perturbations in thickness and velocity show a drastic change in behavior. A steep increase in velocity occurs when the ice thickness decreases $(\omega t=\pi / 2)$, which corresponds to a large increase in flux to facilitate the mass loss. Similarly, when the sheet grows $(\omega t=3 \pi / 2)$, the flux needs to reduce to facilitate growth.

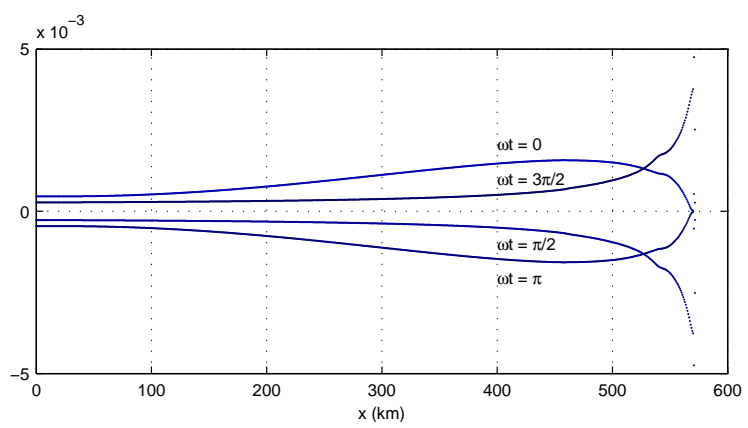

Fig. 10 Oscillating perturbation in the bedrock, given by the eigenvector corresponding to the complex conjugate eigenpair at the Hopf bifurcation.

In the ablation region the perturbation in ice thickness shows a few peculiar oscillations. At the grounding line the sheet thickness seems to be slightly less than at the ice divide, perhaps to facilitate the appropriate flux. However, just before the grounding line and after $x \approx 476 \mathrm{~km}$ the effect of a reverse accumulation feedback seems to be visible. Due to the negative accumulation the bedrock is adjusted differently, see Figure 10. In the oscillatory bedrock perturbation there seems to be some irregular influence from the free boundary. Nevertheless, a change in adjustment around $x \approx 476 \mathrm{~km}$ is visible in at least two phases of the oscillation at $\omega t=0$ and $\omega t=\pi$.

\section{Summary and Discussion}

From the theories of ice-age cycles, it is clear that ice-sheet dynamics plays a central role in the explanation on how the variations in insolation lead to multi-millennial variability of the climate system, in particular on the $100 \mathrm{kyr}$ time scale. A problem with the current theories is that there are many different conceptual models which can give a dominant $100 \mathrm{kyr}$ variability, but it is difficult to falsify them based on the proxy data record [21].

A step forward is to determine spatial patterns of variability associated with the glacial cycles, similar to what has been done for other problems of climate variability such as El Niño [32] and the Atlantic Multidecadal Variability [33]. This obviously requires a next level of models in the hierarchy (see Chapter 6 of [34]), at 
least formulated in terms of partial differential equations (spatially extended models), also (often) referred to as intermediate complexity models (ICMs).

In this contribution, we applied techniques of numerical bifurcation theory to study the bifurcation behavior of solutions of such an ICM two-dimensional ice sheet model [24]. The complication arises here from the grounding line dynamics at $x=x_{g}$, which is in principle a free boundary problem. Here, it is handled by using a transformation $z=x / x_{g}$, where the original domain $x \in\left[0, x_{g}\right]$ is mapped onto the fixed domain $z \in[0,1]$.

In the version of the model where the bottom topography is fixed, the results provide insight into the mechanism of marine ice sheet instability. There are robust intervals in parameter space, where multiple equilibria occur, corresponding to a large ice sheet and a small ice sheet. Hence, for these parameter values, transitions can occur where the ice-sheet decreases substantially in shape and length. Tracing unstable equilibria and performing a stability analysis enables the investigation of the actual structure of the growing perturbation. We have shown numerically that a positive eigenvalue is associated with the instability criterion [29] for the full problem, and that the advected thickness perturbation (the term $\bar{u} \hat{h}$, where $\bar{u}$ is the steady state velocity and $\hat{h}$ the ice thickness perturbation) dominates the instability process.

Transition behavior under the effects of noise in the accumulation parameter $a$ on the grounding line motion under stable conditions have been studied in [35]. The magnitude (for typical noise levels) of these motions is in the order of $1000 \mathrm{~m}$, which are similar amplitudes as observed for example in [36]. It appears to be more likely to jump from a large ice sheet state to a small ice sheet state than vice versa. Grounding line flux variability shows a related asymmetry, likely due to differences in local bedrock conditions and/or global ice sheet extent.

When the load-accumulation feedback is included by extending the ice-sheet with a dynamical bottom topography, oscillatory instabilities occur through a Hopf bifurcation. Here, the eigenvectors associated with the instability provide an interesting spatial pattern of variations of the ice sheet, with largest amplitudes near the grounding line. The time scale here is connected to the relaxation time scale $\tau_{a}$ of the bottom topography reflecting the interaction between the ice sheet and bedrock below.

Of course, such an analysis does not solve the glacial - interglacial variability problem because the ICM used here does not capture essential processes. Currently, this land-ice model, a sea ice model and a carbon cycle model are coupled to the fully implicit ocean model THCM [37, 38] to provide a fully implicit climate model (still very idealized with respect to state-of-the-art global climate models) with which bifurcation analysis can be performed. Using this model, we aim to show that there are different Hopf bifurcations involved in the glacial - interglacial problem and how the external M-forcing can interact with the internal variability generated by these instabilities.

Acknowledgements TEM and HAD acknowledge support by the Netherlands Earth System Science Centre (NESSC), financially supported by the Ministry of Education, Culture and Science (OCW), Grant no. 024.002.001. FWW acknowledge support from the Mathematics of Planet Earth 
research program, project number 657.000.007, which is financed by the Netherlands Organisation for Scientific Research (NWO).

\section{References}

1. L.E. Lisiecki, M. Raymo, Paleoceanography 20(1), PA1003 (2005)

2. J. Jouzel, V. Masson-Delmotte, O. Cattani, G. Dreyfus, S. Falourd, G. Hoffmann, B. Minster, J. Nouet, J.M. Barnola, J. Chappellaz, H. Fischer, J.C. Gallet, S. Johnsen, M. Leuenberger, L. Loulergue, D. Luethi, H. Oerter, F. Parrenin, G. Raisbeck, D. Raynaud, A. Schilt, J. Schwander, E. Selmo, R. Souchez, R. Spahni, B. Stauffer, J.P. Steffensen, B. Stenni, T.F. Stocker, J.L. Tison, M. Werner, E.W. Wolff, Science 317(5839), 793 (2007)

3. J. Imbrie, K.P. Imbrie, Ice Ages: Solving the Mystery (2nd Edn., Harvard Univ. Press, Cambridge, Mass., 1986)

4. M. Milankovitch, in Handbuch der Klimatologie, ed. by W. Koppen, R. Geiger (Borntraeger, Berlin, 1930)

5. M.E. Raymo, P. Huybers, Nature 451(7176), 284 (2008)

6. M. Ghil, Physica D-Nonlinear Phenomena 77, 130 (1994)

7. D. Paillard, Nature 391(6665), 378 (1998)

8. R. Benzi, G. Parisi, A. Sutera, A. Vulpiani, Tellus 34, 10 (1982)

9. R. Benzi, G. Parisi, A. Sutera, A. Vulpiani, SIAM Journal on Applied Mathematics 43, 565 (1983)

10. C. Nicolis, Tellus 34(1), 1 (1982)

11. M. Ghil, S. Childress, Topics in Geophysical Fluid Dynamics: Atmospheric Dynamics, Dynamo Theory, and Climate Dynamics (Springer-Verlag, Berlin/Heidelberg/New York, 1987)

12. H. Le Treut, J. Portes, J. Jouzel, M. Ghil, Journal of Geophysical Research 93, 9365 (1988)

13. M. Ghil, H. Le Treut, Journal of Geophysical Research 86, 5262 (1981)

14. B. Saltzmann, Dynamical Paleoclimatology (Academic Press, 2001)

15. D. Paillard, F. Parrenin, Earth and Planetary Science Letters 227(3), 263 (2004)

16. A.W. Omta, G.A. K. van Voorn, R.E. M. Rickaby, M.J. Follows, Global Biogeochemical Cycles 27(3), 692 (2013). DOI 10.1002/gbc.20060. URL http://dx.doi.org/10.1002/gbc.20060

17. M. Crucifix, Philosophical Transactions Of The Royal Society A-Mathematical Physical And Engineering Sciences 370(1962), 1140 (2012)

18. H. Gildor, E. Tziperman, Paleoceanography 15, 605 (2000)

19. H. Gildor, E. Tziperman, Journal of Geophysical Research 106, 9117 (2001)

20. E. Tziperman, M.E. Raymo, P. Huybers, C. Wunsch, Paleoceanography 21(4), PA4206, (2006)

21. M. Crucifix, G. Lenoir, T. Mitsui, in Nonlinear and Stochastic Climate Dynamics, ed. by C. Franzke, T.J. O'Kane (Cambridge University Press, 2016), pp. 5-20

22. R. Greve, H. Blatter, Dynamics of ice sheets and glaciers (Springer, Berlin, 2009)

23. C.J. Van der Veen, Fundamentals of Glacier Dynamics (CRC Press, 2013)

24. C. Schoof, J. Geophys, Res. 112(F3) (2007). DOI 10.1029/2006JF000664

25. F. Pattyn, C. Schoof, L. Perichon, R.C.A. Hindmarsh, E. Bueler, B. de Fleurian, G. Durand, O. Gagliardini, R. Gladstone, D. Goldberg, G.H. Gudmundsson, V. Lee, F.M. Nick, A.J. Payne, D. Pollard, O. Rybak, F. Saito, A. Vieli, The Cryosphere Discussions 6(1), 267 (2012). DOI 10.5194/tcd-6-267-2012. URL http://www.the-cryosphere-discuss.net/6/267/2012/

26. A. Vieli, A.J. Payne, Journal of Geophysical Research: Earth Surface 110(F1) (2005). DOI 10.1029/2004JF000202. URL http://dx.doi.org/10.1029/2004 JF000202

27. H.A. Dijkstra, Nonlinear physical oceanography: A dynamical systems approach to the large scale ocean circulation and El Niño, vol. 28 (Springer, Berlin, 2005) 
28. Y.A. Kuznetsov, Elements of applied bifurcation theory, Applied Mathematical Sciences, vol. 112, 3rd edn. (Springer-Verlag, New York, 2004). DOI 10.1007/978-1-4757-3978-7. URL http://dx.doi.org/10.1007/978-1-4757-3978-7

29. C. Schoof, J. Fluid Mech. 698, 62 (2012). DOI 10.1017/jfm.2012.43

30. J. Van den Berg, R. Van de Wal, J. Oerlemans, Journal of Glaciology 52(176), 89 (2006). URL http://www.ingentaconnect.com/content/igsoc/jog/2006/00000052/00000176/art00009

31. M. Ghil, Physica D: Nonlinear Phenomena 77, 130 (1994)

32. H.A. Dijkstra, G. Burgers, Annual Review of Fluid Mechanics 34, 531 (2002)

33. L.M. Frankcombe, A. von der Heydt, H.A. Dijkstra, Journal Of Climate 23(13), 3626 (2010). Doi: 10.1175/2010JCLI3471.1

34. H.A. Dijkstra, Nonlinear Climate Dynamics (Cambridge University Press, 2013)

35. T.E. Mulder, S. Baars, , F. Wubs, H.A. Dijkstra, J. Fluid Mechanics (2018). In press

36. A.E. Hogg, A. Shepherd, N. Gourmelen, M. Engdahl, Journal of Glaciology 62(236), 1104 (2016)

37. A.C. De Niet, F. Wubs, A.D. Terwisscha van Scheltinga, H.A. Dijkstra, J. Comp. Phys. 277, 654 (2007)

38. J. Thies, F. Wubs, H.A. Dijkstra, Ocean Modelling 30(4), 287 (2009) 